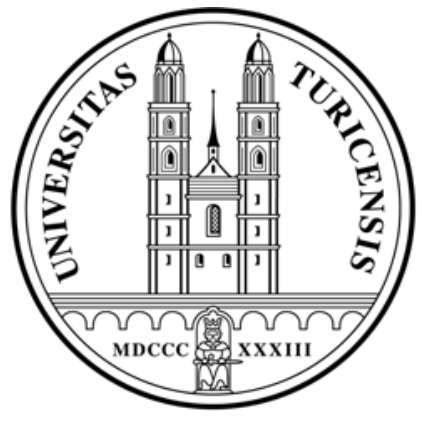

Institute for Empirical Research in Economics

University of Zurich

Working Paper Series

ISSN 1424-0459

Working Paper No. 507

\title{
Inequality and Growth: The Neglected Time Dimension
}

Daniel Halter, Manuel Oechslin and Josef Zweimüller

Revised version, November 2011 


\title{
Inequality and Growth: The Neglected Time Dimension
}

\author{
Daniel Halter, \\ Manuel Oechslin, \\ University of Zurich \\ University of Bern* \\ Josef Zweimüller, \\ University of Zurich and CEPR ${ }^{\dagger}$
}

November 18, 2011

\begin{abstract}
The empirical literature on the relationship between inequality and growth offers a contradictory assessment: Estimators based on time-series variation indicate a positive link while estimators (also) exploiting the cross-sectional variation suggest a negative relationship. The present paper (i) confirms this conflicting pattern in an expanded dataset; (ii) proposes a simple theoretical framework to highlight the biases associated with the different techniques. We argue that mechanisms generating a positive inequality-growth relationship work mainly in the short-run and are reflected in difference-based estimators. In contrast, mechanisms generating a negative relationship work over the longer term and are reflected in level-based estimators. (JEL O11, O15, O43, C23)
\end{abstract}

Keywords: Inequality, growth, short-run effects, long-run effects

${ }^{*}$ Corresponding author. University of Bern, World Trade Institute, Hallerstrasse 6, CH-3012 Bern, Switzerland; phone: +413163136 73; fax: +413163136 30; email: manuel.oechslin@wti.org.

${ }^{\dagger}$ University of Zurich, Department of Economics, Mühlebachstrasse 86, CH-8008 Zürich; phone: +41 4463437 24; e-mail: josef.zweimueller@econ.uzh.ch. 


\section{Introduction}

Over the past two decades, theoretical work has come up with a substantial number of channels through which inequality may affect economic growth, either in a positive or in a negative direction. These theoretical contributions have made clear that the impact of inequality is quite complex and likely to depend on, among other things, the specifics of a country (e.g., the stage of economic development; the extent of market failures; the form of government) or the time horizon considered (e.g., short run vs. long run). This ambiguity is also mirrored in the empirical literature which finds both significantly positive and negative effects, and sometimes no effects at all.

Figure 1 here

Yet, a closer look at the empirical literature reveals an interesting pattern. Estimates based on time-series variation only (e.g., estimations relying on fixed-effects or first-differences estimators such as those in Li and Zou, 1998; Forbes, 2000) consistently find a strong positive impact of inequality on subsequent growth. On the other hand, estimation methods which also or exclusively exploit the cross-sectional variation in the data tend to find a negative relationship. Examples in this regard include Barro's (2000) random-effects approach and earlier studies based on simple cross-country OLS estimates (e.g., Alesina and Rodrik, 1994; Persson and Tabellini, 1994; Deininger and Squire, 1998; Clarke, 1995). These results in the literature can already be seen from a look at some crude data. Panel $a$. of Figure 1 is based on time-series variation only. Exploiting multiple observations within countries, it plots changes in the log GDP per capita (p.c.) against changes in the lagged Gini coefficient and reveals a mildly positive relationship. Panel b. highlights the relationship in levels. It plots the log GDP p.c. against the lagged Gini coefficient and documents a clear negative link. ${ }^{1}$

This paper contributes to the literature in two ways. First, we show that the pattern of existing results is indeed driven by the choice of methods rather than idiosyncratic

\footnotetext{
${ }^{1}$ Figure 1 is about inequality and GDP p.c., both in terms of first differences $(a$.$) and levels (b$. $)$. It is this variation that is exploited in the GMM estimation below (see equations 1 and 2 of Section 2.1).
} 
differences across studies (such as the selection of countries, time periods, or included control variables). We do so by taking advantage of an expanded and more comprehensive inequality dataset. Also in this much larger dataset, the first-differences GMM estimator consistently finds a strong positive inequality-growth relationship while the system GMM estimator (which also exploits the cross-sectional variation) identifies a strong negative link in a broad set of poorer countries (and a positive link in just a small number of high-income countries). Second, we interpret these results through the lens of the recent theoretical literature on economic growth and development. We argue that the standard regression equation underlying most empirical estimates is (mis-)specified in a way that induces (i) the first-difference GMM estimator to systematically pick up the positive (short-run) effects of inequality; (ii) the system GMM estimator to reflect primarily the negative (long-run) consequences. In other words, the paper's second contribution is to highlight and interpret the systematic biases associated with the standard approaches rather than coming up with an unbiased estimator.

To convey our argument in a clear and concise way, we introduce a simple model which reflects that the positive and negative effects of inequality cluster in a specific way. In the theoretical literature, inequality is said to promote growth by fostering aggregate savings (Kuznets, 1955; Kaldor, 1955); by promoting the realization of high-return projects (Rosenzweig and Binswanger, 1993); or by stimulating R\&D (Foellmi and Zweimueller, 2006). On the other hand, inequality is expected to hamper growth by promoting expensive fiscal policies (Perotti, 1993); by inducing an inefficient state bureaucracy (Acemoglu et al. 2008); by hampering human capital formation (Galor and Zeira, 1993; Galor and Moav, 2004); by leading to political instability (Bénabou, 1996); or by undermining the legal system (Glaeser et al., 2003). Most of the positive effects (e.g., those operating through convex savings functions, market imperfections or innovative incentives) rely on purely economic mechanisms. Arguably, these effects materialize relatively fast, in the short or medium run. Most of the negative effects, however, involve the political process, the change of institutions, the rise of socio-political movements, or they operate through changes in educational attainment of the population. Most likely, these effects take time and materialize primarily in the long run. 
Based on these arguments, the contradictory evidence on the inequality-growth relationship can be reconciled in a natural way. Studies that exploit mainly the time-series dimension of the data, such as the first-differences GMM estimator, regress changes in (log) output on (slightly) lagged changes in inequality. When inequality goes up, the positive short- or medium-run effects are associated with positive changes in inequality while the subsequent negative changes (i.e., those coming from the long-run effects) are treated as noise. Thus, the first-differences estimator only reflects the positive short- or medium-run effects but leaves out the adverse long-run consequences (see Figure 1a.).

In contrast, the system GMM estimator is likely to find a negative relationship, in particular if (i) the negative long-run effects dominate the positive short- or medium-run effects (which is more likely to be the case in poorer economies); (ii) if inequality is highly persistent (which is actually true everywhere). Under these circumstances, the majority of observations is either of the type "low level of inequality and high level of output" or "high level of inequality and low level of output." Hence, the system GMM estimator (which also exploits the cross-country variation) tends to find a negative relationship (see Figure 1b.) - which reflects the overall adverse impact of inequality.

The present paper is part of a small literature which tries to get a better grasp of the empirical picture with respect to the inequality-growth relationship. Earlier contributions include Banerjee and Duflo (2003) and Voitchovsky (2005). The former paper presents evidence suggesting that changes in inequality (in any direction) are associated with reduced growth in the short run; as a result, the standard regression equation might be mis-specified in a way that - misleadingly - makes differences-based estimators indicate a positive relationship. Voitchovsky (2005), by contrast, argues that inequality coming from the top end of the income distribution is indeed likely to promote economic growth while bottom-end inequality tends to be harmful. She therefore suggests controlling separately for inequality coming from different parts of the distribution (and finds supportive evidence in a panel of rich countries). None of these papers, however, focuses specifically on the time dimension, and so we view this paper as complementary. Finally, on a broader level, our study contributes to a growing body of research (e.g., Hauk and Wacziarg, 2009) on the bias properties of standard estimation methods that are used in 
the empirical growth literature.

The remainder of this paper is organized as follows. Section 2 presents the empirical results and links them to the earlier literature. In Section 3, we introduce a model to interpret our findings through the lens of the theoretical literature. Section 4 concludes.

\section{Empirical Results}

We now apply the standard estimators to a common dataset, relying on a common set of controls. We find the inequality-growth relationship to be consistently positive when we rely on time-series variation only. However, when we also exploit the cross-sectional variation, the relationship turns significantly negative in a broad sample of poorer countries. This suggests that the pattern of existing results is driven by the choice of methods rather than idiosynchratic differences across studies (such as the selection of countries, time periods, or control variables).

\subsection{Specification and Estimation}

Specification and data. We rely on a standard 5-year panel data model which is similar to those used in several recent empirical studies on growth (e.g., Caselli et al., 1996; Barro, 2000; Forbes, 2000; Voitchovsky, 2005). Specifically, we estimate

$$
y_{i t}-y_{i t-1}=\gamma y_{i t-1}+\mathbf{x}_{i t}^{\prime} \boldsymbol{\delta}+\zeta_{t}+\eta_{i}+v_{i t}
$$

where $i=1, \cdots, N$ denotes a particular country and $t=1, \cdots, T$ is time (with $t$ and $t-1$ five years apart). The variable $y$ stands for the log of real GDP p.c. so that the left-hand side of equation (1) approximates country $i$ 's five-year growth rate in the years between $t-1$ and $t$. On the right-hand side, we have $y_{i t-1}$ to control for convergence; a vector $\mathbf{x}_{i t}$ consisting of variable country characteristics; a period-specific effect $\zeta_{t}$ to capture productivity changes common to all countries; a country-specific effect $\eta_{i}$ to capture timeinvariant and unobserved country characteristics; an idiosyncratic error term $v_{i t}$.

The vector $\mathbf{x}_{i t}$ consists of the Gini index and three additional standard control vari- 
ables. In line with the recent literature (e.g., Perotti, 1996; Forbes, 2000), these additional variables are the average years of secondary schooling in the population aged over 25 (separately for males and females) and the price level of investment (to control for market distortions). In general, the explanatory variables are measured at the beginning of each 5 -year period. In case of inequality, this is not always possible because the Gini index is not usually available on an annual basis. In these cases, we take the last available value in the previous 5-year period.

The analysis includes up to 90 countries and covers the period from 1966 to 2005. The GDP per capita data comes from the World Development Indicators (World Bank, 2006) and is in constant 2000 US\$. The Deininger and Squire (1996) data base serves as the primary source for the inequality data. However, in order to broaden our sample in the cross-sectional as well as the time-series dimension, we also rely on a subsidiary source, the UNU-WIDER (2008) data base. ${ }^{2}$ Finally, the education data comes from Barro and Lee (2001) and the source for the price of investment is Heston et al. (2006; PWT 6.2). More detailed sources and definitions, as well as summary statistics, are presented in Table 1.

Table 1 here

Estimation methods. It is well-known that the standard panel data methods (i.e., fixed-effects $[\mathrm{FE}]$ and random-effects $[\mathrm{RE}]$ estimations) are unlikely to provide consistent estimates of $\gamma$ and $\boldsymbol{\delta}$ (see, e.g., Bond et al., 2001). Obviously, using the RE estimator is problematic because the unobserved country effect, $\eta_{i}$, is presumably correlated with the other explanatory variables. A second problem emerges when we rewrite model (1) as

$$
y_{i t}=(\gamma+1) y_{i t-1}+\mathbf{x}_{i t}^{\prime} \boldsymbol{\delta}+\zeta_{t}+\eta_{i}+v_{i t}
$$

Equation (2) highlights that controlling for convergence in a panel data growth model introduces a lagged dependent variable. As a result, even if equations (1) and (2) gave an accurate description of reality, both the RE estimator and the FE estimator would be

\footnotetext{
${ }^{2}$ At the end of Table 1, we describe how our inequality dataset is constructed and how some pitfalls associated with the use of secondary datasets (see Atkinson and Brandolini, 2001) have been addressed.
} 
very likely to give inconsistent estimates of the parameters $\gamma$ and $\boldsymbol{\delta}$.

To deal with these problems, the literature has developed specific GMM estimation techniques, most notably the first-difference GMM estimator and the system GMM estimator. The first-difference GMM estimator was developed by Arellano and Bond (1991) and is similar to the FE estimator in the sense that it exploits only within-country variation. The idea is to eliminate the country-specific effect by differencing model (2) and then to use sufficiently lagged values of $y$ and $\mathbf{x}$ as instruments. However, although the first-difference GMM estimator "solves" the problems of unobserved heterogeneity and lagged dependent variables, it has been criticized for the fact that it does not make use of the variation in levels. The main concern is that the cross-sectional variation embodies a large part of the information since within-country inequality is quite persistent. ${ }^{3}$ Thus, ignoring this cross-sectional variation may give rise to unnecessarily large biases and imprecision. One way to address these shortcomings is to use the system GMM estimator pioneered by Arellano and Bover (1995) and Blundell and Bond (1998). While requiring a more stringent set of restrictions, ${ }^{4}$ the system GMM procedure does better in terms of efficiency since - like the RE estimator - it also exploits the cross-country variation in the data (see, e.g., Bond et al., 2001, for the details).

In what follows, we will apply both GMM estimation techniques to our expanded dataset and document that - consistent with the existing empirical picture - the two approaches may lead to systematically different estimation results. Section 3 is then devoted to explaining these differences across methods with the help of a simple model.

\subsection{Results}

Time-series variation only. We now go through the first-difference estimation results. To connect with the previous literature, we first present evidence based on a sample which is similar to that in Forbes (2000) in terms of countries included and periods covered. We

\footnotetext{
${ }^{3}$ This observation also applies to our dataset: The adjusted $R^{2}$ from a regression of the Gini coefficient on country dummies is 0.84 (and rises only to 0.85 if time dummies are also included).

${ }^{4}$ For the instruments used by this estimator to be valid, the Blundell-Bond (1998) requirement must be satisfied. In the context of growth regressions, this means that there must not be a systematic link between a country's fixed effect and its distance from the steady state (see Roodman, 2009).
} 
then show that these results are quite robust to the inclusion of additional countries and more recent observations as well as to a number of other modifications.

The first column of Table 2 gives the results based on the Forbes sample (which includes 42 countries and covers the 1965-1995 period). Like Forbes, we find a significant positive impact of inequality on growth, and the magnitude of the effect is very similar: On an annualized basis, our estimates imply a coefficient of 0.0015 while Forbes (2000) reports one of 0.0013 . As the second column shows, the coefficient on inequality remains significant and comparable in size after extending the sample by two additional 5-year periods (i.e., the 1996-2000 and 2001-2005 periods). Similarly, as documented in the third column, the inclusion of 28 additional countries does not change the basic empirical finding: Higher inequality has a significantly positive impact on (short-run) growth, albeit the effect is somewhat smaller in the broader country sample (which includes a larger fraction of less-advanced countries). ${ }^{5}$

\section{Table 2 here}

The remaining columns of Table 2 document the robustness of this empirical outcome to some natural variations. First, the estimates in columns (4) and (5) are based on subsets of the full sample. Specifically, column (4) shows the impact of inequality in countries which are classified as high income or upper-middle income (according to the 2009 World Bank definition); column (5) provides the corresponding results for the remaining countries (lower-middle income or low income). Apparently, although the two subsets contain very different economies, the estimated impact of inequality is still significantly positive in both cases and also of very similar size across the two country groups.

The second modification concerns the time structure of the panel. In order to check whether the above results are not just an artifact of the 5-year structure, the estimates in columns (6) and (7) are based on four 10-year periods. The results suggest that higher inequality tends to foster growth also over this medium time horizon, and the size of the

\footnotetext{
${ }^{5}$ Note that 20 of these 28 additional countries are low income or lower-middle income countries according to the classification by the World Bank (2006). As a result, in the full sample, $47 \%$ of the countries fall into these two categories (while the rest belong to the categories upper-middle or high income).
} 
estimated impact is somewhat larger: For instance, on an annualized basis, the coefficient in the fourth column (5-year periods; high and up-mid countries) is 0.00082 while the corresponding coefficient for the 10-year structure is 0.00114 . However, the estimates are less precise - which is not surprising given the much smaller number of observations.

The validity of the first-difference estimator depends on the absence of serial correlation in the error terms, $v_{i t}$. This means that the differenced error terms should not show secondorder serial correlation (though they have a first-order correlation by construction). The statistics $M 1$ and $M 2$ in Table 2 give the $t$-values associated with the tests for, respectively, first-order and second-order correlation in the $\triangle v_{i t}$-series. As the numbers show, serial correlation may only be an issue in the first regression (Forbes replication) but not in columns $(2)-(7)$. Finally, the high $p$-values on the Hansen test also indicate that the joint validity of the instruments cannot be rejected. Yet, $p$-values close to 1 suggest that there might be a problem of "too many instruments" (as discussed in Roodman, 2009). We will return to this issue below.

Time-series and cross-sectional variation. Table 3 presents the results based on the system GMM estimator. The first column presents the estimates based on the full sample. Unlike in all the regressions shown in the previous table, the estimated impact of inequality on growth is now negative, yet not significantly so. ${ }^{6}$ More precise results can be gained by splitting the country sample along income classes (columns $2-4$ ). It turns out that, as shown in the second column, the system GMM estimates also indicate a positive impact of inequality among the small group of high-income countries. However, there is no significant relationship among upper-middle income countries (third column) ${ }^{7}$ and - most importantly - the system GMM estimates indicate a negative impact in the large group of countries with lower-middle income or low income (fourth column). Note further that switching to a 10-year structure again confirms the results obtained under

\footnotetext{
${ }^{6}$ Note that the number of countries in the sample increases from 70 to 90 . The reason is that the first-difference estimator requires at least three consecutive observations of $y$. This is not true in the case of the system GMM estimator which allows for moment conditions stemming from the regression equation in levels: Since there are predetermined variables (lagged values of which can be used as instruments), there exist moment conditions which do not require consecutive observations of $y$.

${ }^{7}$ If we combine - as in Table 2 - high-income countries and upper-middle income countries in one sample, the estimated coefficient on inequality is insignificant (result not reported in the table).
} 
the 5 -year structure (columns 6 and 7 of Table 3 ).

Table 3 here

So, even though the test statistics at the bottom of Table 3 support the validity of the instruments with this estimation strategy too, the system GMM approach paints a decidedly different picture than the first-difference estimator: While the latter uniformly points to a positive relationship (and thus confirms the results of, e.g., Li and Zou, 1998; Forbes, 2000), the findings here suggest that the impact of inequality on growth is negative (or at least non-positive) in countries which are not among the richest. Note that this result is perfectly in line with Barro's (2000) RE analysis (which also exploits crosssectional as well as time-series variation) and also matches the results in earlier OLS-based studies such as those of Alesina and Rodrik (1994) or Persson and Tabellini (1994).

\subsection{Robustness Issues}

Additional controls. In choosing our explanatory variables, we follow the standard specification used by, among others, Perotti (1996) and Forbes (2000). However, we also explored whether other specifications would change our results. In particular, we ran additional regressions which included further explanatory variables that are used in the empirical growth literature, namely the investment rate and the population growth rate (as, for instance, in Mankiw, Romer, and Weil, 1992). The results are shown in Appendix B, Table 4. The basic picture is that the baseline results reported in Tables 2 and 3 are robust to the inclusion of these additional variables. Regarding the impact of inequality in the full sample, the only notable difference is that system GMM estimator finds a significantly negative impact when the investment rate is added as an explanatory variable (Table 4, column 6). To save space, Table 4 does not show the system GMM estimates for the different country sub-samples. However, we can report that the inclusion of the additional controls does not significantly change the baseline estimates shown in columns (2) to (4) of Table 3 (detailed results available upon request). Consistent with the findings in the full sample, the only difference is that the impact of inequality is 
no longer significantly positive among high-income countries (but remains significantly negative among lower middle- and low-income countries).

Reducing the instrument count. Following the existing literature (e.g., Forbes, 2000), we have not restricted the set of internal instruments relied upon (by default) by the two GMM techniques. Yet, as discussed in Roodman (2009), using an unrestricted set of moment conditions may give rise to a "problem of too many instruments" - which expresses itself in a weakened Hansen test of the instruments' joint validity. To address this problem, we followed Roodman and re-ran our regressions using "collapsed instruments" (see Roodman, 2005, Section V). The result of this exploration is that our baseline findings are fairly robust. When we redo the main first-difference GMM estimation reported in Table 2, which is the one in column (3), we find that the impact of inequality is of about the same size, though the coefficient is no longer significantly different from zero. The $p$-value on the Hansen test drops substantially to 0.12 but still suggests that the joint validity of the instruments cannot be rejected at the $10 \%$ level. Similarly, when we redo the system GMM estimation reported in Table 3, column (2), we find the point estimate to be largely unchanged but no longer significantly different from zero (while the $p$-value on the Hansen test is still 0.961). Finally, when we redo the system GMM estimation in column (4), we continue to find inequality to have a significantly negative impact (and the $p$-value on the Hansen test is 0.46 ).

\section{Interpreting the Empirical Results}

The present section looks at how these seemingly contradictory estimation results can be interpreted and reconciled. We first discuss that, in fact, the existing literature suggests that both relationships should be present in reality. In the second step, we introduce a simple model which summarizes the literature in a parsimonious way. With the help of the model, we then argue that regression equation (1) is mis-specified so that the two different GMM estimators are prone to systematically reflect just one of the two relationships, namely the positive one in the case of the differences-based approach and 
the negative one if the estimator also exploits cross-sectional variation.

\subsection{Short-run and Medium-run Effects vs. Long-run Effects}

Inequality affects growth through many channels, and theoretical work discusses both negative and positive effects. Yet, as the following overview shows, there seems to be clear pattern in the literature: The positive effects rely on purely economic mechanisms and should therefore be expected to set in fast. The negative effects, on the other hand, often involve political-economy arguments; as a result, they may need more time to materialize.

As to the positve channels, the literature has long argued that savings functions tend to be convex in wealth (see, e.g., Kuznets, 1955; Kaldor, 1955). So, other things equal, higher inequality is associated with higher aggregate savings and thus faster convergence to the balanced growth path. More recently, the focus has been on the impact of inequality on the selection of investment projects (see, e.g., Matsuyama, 2000, in particular Section 4). The main argument here is that, if the financial system is imperfect, access to external finance depends on personal wealth. As a result, if wealth is widely spread among the population, nobody may be able to raise sufficient funds to realize high-return projects which require large investments. In this case, a more concentrated distribution of productive assets may put at least a limited number of entrepreneurs into a position to realize such projects - and thus boosts growth. ${ }^{8}$ This effect is reinforced by the fact that the high-return projects are often the more risky ones (see, e.g., Rosenzweig and Binswanger, 1993). As a result, with a relatively equal wealth distribution, the number of entrepreneurs who are sufficiently rich to absorb significant risks may be very small. So, once again, a more concentrated distribution of wealth may multiply the number of high-return projects realized. Finally, the literature also discusses positive demand-side effects. With a more unequal distribution, a larger fraction of total demand falls on "high-end" products (as opposed to goods satisfying basic needs). Thus, innovators benefit from larger home markets which more easily support the investments required to develop novel or better

\footnotetext{
${ }^{8}$ It has also been argued that, with convex technologies and financial markets imperfections, higher inequality deteriorates economic performance because investment returns are more heterogeneous. However, as shown by Foellmi and Oechslin (2008), this is by no means a robust theoretical prediction.
} 
varieties (see, e.g., Foellmi and Zweimueller, 2006).

While working through different channels, these positive effects have one thing in common: They emphasize purely economic mechanisms and therefore tend to materialize fast. This is not true for the negative channels. Most of them rely on political-economy arguments. For instance, it has been pointed out that more unequal societies tend to have higher levels of redistribution and hence higher levels of taxation - which weakens the incentives to save and invest (see, e.g., Perotti, 1993). A related argument focuses on the composition of government expenditures. With higher inequality, the decisive voter supplies fewer production factors (i.e., physical or human capital). As a result, he may strongly prefer direct transfers over productive investments in public goods. Finally, even if the rich hold political power, inequality may still have a negative impact via the fiscal policy channel. As highlighted by Acemoglu et al. (2008), if inequality is high, an oligarchic government may set up an inefficient bureaucracy to avoid high taxation once the country is transformed into a democracy. ${ }^{9}$ Yet, via these channels, changes in inequality cannot be expected to have an immediate effect. It takes time for shifts in policy preferences to be reflected in similar changes within the legislative body. Moreover, even with a fresh legislature in place, altering tax laws (or even changing the bureaucracy) is time consuming. Further negative effects are also unlikely to materialize quickly. If higher inequality reduces spending on education (see, e.g., Galor and Zeira, 1993; Galor and Moav, 2004), it may take a decade for the effects to be felt. Similarly, it may be a long time before disaffection caused by higher inequality is bundled in social movements which then may threaten political stability (see, e.g., Bénabou, 1996) or before higher inequality has undermined the security of property rights (see, e.g., Glaeser et al., 2003).

Note, finally, that many of these negative long-run effects are probably less pronounced in richer economies where technologies are more advanced and democracy tends to be better established. For instance, inequality is less likely to promote political instability in democratic environments which offer broad participation in the political process. Similarly, more advanced technologies are more dependent on an adequate supply of public

\footnotetext{
${ }^{9}$ More generally, based on the experience of the colonization of the New World, Sokoloff and Engerman (2000) argue that huge wealth inequalities may promote institutions that protect the privileges of the elites and restrict opportunities for the broad masses - with adverse consequences for economic development.
} 
goods (i.e., fast enforcement of private contracts; reliable power supply) so that even relatively poor people tend to be cautious when demanding more transfers at the expense of productive public investment.

\subsection{A Formal Approach}

We now present a simple model which includes both a positive short-run effect (i.e., an economic channel) and a negative long-run effect (i.e., a political-economy channel) of inequality. We then use this model to suggest a natural interpretation of the empirical findings in Section 2 and discuss how this interpretation depends on three three crucial magnitudes, the short-run effect, the long-run effect, and the persistence of inequality.

\subsubsection{A Parsimonious Model}

Assumptions. We focus on an infinite-horizon economy which is populated by a continuum of individuals of measure 1. All agents derive utility from consumption of a single (non-storable) output good, and preferences are represented by the utility function

$$
U_{t}=E_{t}\left\{\sum_{s=0}^{\infty} \beta^{s} c_{t+s}\right\},
$$

whereas $c_{t}$ denotes consumption in period $t$. Individuals differ regarding their endowment with the productive asset (which we may interpret as "skills", for instance). A fraction $\alpha>1 / 2$ of the population (the "poor", $P$ ) is endowed with $\omega^{P}\left(D_{t}\right)<1$ units of this asset, whereas 1 is the average endowment in the economy. The endowment of the remaining agents (the "rich", $R$ ) is then given by $\omega^{R}\left(D_{t}\right)=\left(1-\alpha \omega^{P}\left(D_{t}\right)\right) /(1-\alpha)>1$. The state variable $D_{t} \in\{L, H\}$ represents the degree of inequality, whereas $L$ stands for low inequality so that $\omega^{P}(L)>\omega^{P}(H)$. Note further that, at the beginning of each period, inequality may change exogenously. In particular, we have $D_{t}=D_{t-1}$ with probability $\pi$ and $D_{t} \neq D_{t-1}$ with probability $1-\pi$. Thus, a high value of $\pi$ mirrors strong persistence in inequality. In practice, a change in the distribution of skills may be due to a shock to the educational system which improves the quality of primary education relative to that of university education, for instance. 
Suppose further that the individuals have access to a linear technology of the form

$$
y^{i}\left(D_{t}, G_{t}\right)=a^{i} \omega^{i}\left(D_{t}\right) X\left(G_{t}\right)
$$

with $i \in\{P, R\}$, whereas $a^{i}$ is a group-specific productivity parameter and $X\left(G_{t}\right)$ denotes the level of the public good provided by the government. Rich agents are assumed to be more productive than the poor: $a^{R}>a^{P}$. A natural way to think of this assumption is that the more productive technology requires a certain skill level which cannot be achieved by the poor. ${ }^{10}$ The state variable $G_{t} \in\{0,1\}$ reflects whether - in the previous period - the government has invested in the public good, with 1 indicating investment. Hence, $X(1)-X(0) \equiv \triangle X>0$.

On the aggregate level, we can now infer that (private-sector) output is given by

$$
Y\left(D_{t}, G_{t}\right)=\left(a^{R}-\alpha\left(a^{R}-a^{P}\right) \omega^{P}\left(D_{t}\right)\right) X\left(G_{t}\right) .
$$

Other things equal, $Y$ is higher in the high-inequality state $\left(D_{t}=H\right)$ since a larger fraction of the productive asset is allocated to the high-return technology; similarly, output is higher if the level of the public good is high $\left(G_{t}=1\right)$. In what follows, we impose

$$
\frac{X(1)-X(0)}{X(1) \omega^{P}(L)-X(0) \omega^{P}(H)}>\alpha \frac{a^{R}-a^{P}}{a^{R}},
$$

so that $Y(L, 1)>Y(H, 0)$. This condition ensures that the long-run effect of inequality is quantitatively more important than the short-run effect.

Turning to the public sector, suppose that the government has access to an income stream of $Z$ units of the final good. We can think of this income as arising from a publicly owned enterprise, the natural resource sector, etc. Regarding public spending, the government has to decide on $G_{t+1}$ in each period $t$. A decision to invest is associated with a contemporaneous cost of $F<Z$ units of the final good. The budget surplus is distributed to the population in a lump-sum manner. Finally, when deciding on $G_{t+1}$, we

\footnotetext{
${ }^{10}$ More generally, this assumption can be seen as a reduced-form representation of the notion that only relatively rich people can rely on high-return technologies because - as discussed in Subsection 3.1 - the financing of such technologies requires good access to the financial system (which the poor lack).
} 
assume that the government has no choice but to implement the variant preferred by the majority of the population, i.e., the poor.

Equilibrium. We now describe the different possible equilibrium patterns of public investment and discuss which pattern emerges under what parameter constellation.

Proposition 1 (i) The politico-economic equilibrium shows fluctuations in the provision of the public good, with a positive level of investment in times of low inequality (i.e., $G_{t+1}=1$ if $\left.D_{t}=L\right)$ and no investment in times of high inequality (i.e., $G_{t+1}=0$ if $\left.D_{t}=H\right)$, if

$$
\frac{\triangle X}{F} a^{P}\left(\pi \omega^{P}(L)+(1-\pi) \omega^{P}(H)\right) \geq \frac{1}{\beta}>\frac{\triangle X}{F} a^{P}\left(\pi \omega^{P}(H)+(1-\pi) \omega^{P}(L)\right) .
$$

Otherwise, the public good is always provided (if $1 / \beta$ is smaller than the last expression in 7) or never provided (if $1 / \beta$ is bigger than the first expression in 7 ).

(ii) More advanced economies (as proxied by the productivity parameter $a^{P}$ ) are more likely to experience an invariably positive supply of the public good, other things equal.

\section{Proof. See Appendix A.}

Intuitively, when condition (7) holds, the poor prefer direct transfers over public investment if inequality is high: High inequality means that the poor can gain little from the public good since they own only a small fraction of the productive asset. In the case of low inequality, however, this gain is sufficiently strong to make the poor prefer investment over higher lump-sum transfers. On the other hand, if condition (7) is violated, the gain from public investment is such that the poor consistently favor or oppose the provision of the public good. Finally, the proposition also highlights that access to more productive technologies makes the poor more likely to consistently favor the provision of the public good (over higher transfers).

Inequality and output. The link between inequality and output is most involved if condition (7) holds. Then, the prediction is that an increase in inequality leads to a short-run increase and a long-run fall in output. Corollary 1 discusses the associated 
co-movements of inequality and output in terms of changes. Corollary 2 looks at the relationship in levels.

Corollary 1 Suppose that conditions (6) and (7) hold. Moreover, assume that inequality has been unchanged between periods $t-2$ and $t-1$. Then,

(i) a rise in inequality in t leads to a contemporaneous rise in output: $Y_{t-1}=Y(L, 1)<$ $Y_{t}=Y(H, 1)$; in $t+1$, output falls, with inequality either unchanged or decreasing.

(ii) a fall in inequality in $t$ leads to a contemporaneous fall in output: $Y_{t-1}=Y(H, 0)>$ $Y_{t}=Y(L, 0)$; in $t+1$, output rises, with inequality either unchanged or increasing.

The intuition behind Corollary 1 is that the level of the public good is a state variable and thus cannot change quickly. So an increase in inequality must lead to a positive effect on output in the short run (i.e., with $X$ still at the high level) but to a negative one in the long run (i.e., when the rise in inequality has undermined to supply of the public good).

Corollary 2 Suppose that the conditions (6) and (7) hold. Moreover, assume that inequality is persistent (i.e., that $\pi$ is "high"). Then, over time,

(i) a large fraction of the observations $\left(D_{t}, Y_{t}\right)$ will either be "low" inequality and "high" output, $(L, Y(L, 1))$, or "high" inequality and "low" output $(H, Y(H, 0))$.

(ii) very few observations $\left(D_{t}, Y_{t}\right)$ will either be "low" inequality and "very low" output, $(L, Y(L, 0))$, or "high" inequality and "very high" output, $(H, Y(H, 1))$.

The central point behind Corollary 2 is persistence in inequality. Persistence means that periods with changes in inequality - which generate observations of the type ("high" inequality/"very high" output) or ("low" inequality/"very low" output) - are infrequent.

The link between inequality and output is much simpler if condition (7) is violated. Then, the supply of the public good is constant over time so that "high" inequality is always associated with "high" output and "low" inequality with "low" output (similarly, a rise in inequality is always associated with an increase in output, and vice versa).

\subsubsection{Reconciling Differences- vs. Level-based Estimates}

We now demonstrate that, if public investment fluctuates, the estimated sign of the inequality-output relationship depends on the estimation method (while it is invariant if 
public investment is stable). We then interpret the empirical results in Section 2 through the lens of this model.

Figure 2 here

Fluctuating supply of the public good. Suppose that the supply of the public good fluctuates, a situation which - according to Proposition 1 - is more likely to emerge in poorer than in richer economies. To demonstrate the decisive role of the estimation method under these circumstances, we combine the information in Corollaries 1 and 2 in a single picture, Figure 2. To see how the figure is constructed, consider an increase in inequality in period $t$. If the focus is on changes (Panel $a$.), the following observations are generated: Observation $-1^{i n}$ in period $t-1$, observation $0^{\text {in }}$ in period $t$ (when the short-run effect materializes), and - in period $t+1$ - observation $1_{a}^{\text {in }}$ (if $D$ is unchanged in $t+1$ so that only the long-run effect materializes) or observation $1_{b}^{\text {in }}$ (if $D$ decreases in $t+1$ so that the long-run effect materializes together with a negative short-run effect). The remaining observations in Panel $a$. can be generated by going through the opposite case, i.e., by considering a decrease in inequality in period $t$. Note that the numbers in Panel $b$. refer to the same thought experiments, but from the perspective of the levels. The two panels further indicate the theoretical frequencies with which the different types of observations occur.

Figure 2 illustrates that the different aspects of the relationship between inequality and output are picked up by different estimation methods. If the relationship is assessed on the basis of changes (Panel $a$.), we can see that estimating a linear regression would give us a clear positive relationship. On the other hand, if levels are considered (Panel b.), fitting a linear trend line would arguably point to a significant negative impact of inequality (since the observations marked by a bigger dot are much more numerous than the remaining observations).

We now establish analytically how close the different estimation methods come in identifying the different aspects of the inequality-output relationship. We start by deriving the formal relationship between output and inequality, assuming that conditions (6) and 
(7) hold. Taking logs on both side of equation (5) gives us

$$
y_{t} \equiv \ln Y_{t}=\ln \left(1-\alpha \frac{a^{R}-a^{P}}{a^{R}} \omega^{P}\left(D_{t}\right)\right)+\ln \left(1+\frac{\triangle X}{X(0)} \frac{H-D_{t-1}}{H-L}\right)+\ln a^{R}+\ln X(0),
$$

whereas the second term on the right-hand side represents the equilibrium expression for $X\left(G_{t}\left(D_{t-1}\right)\right)$. Assume now further that $\omega^{P}\left(D_{t}\right)=1-D_{t}$ so that $D_{t} \in\{L, H\}$ is the difference between the average endowment and the endowment of the poor. Then, the above expression can be approximated by the linear regression equation

$$
y_{i t}=\delta_{1} D_{i t}+\delta_{2} D_{i t-1}+\eta_{i}+v_{i t}
$$

whereas $\delta_{1} \equiv \alpha\left(a^{R}-a^{P}\right) / a^{R}, \delta_{2} \equiv-\triangle X /(X(0)(H-L))$, and $\delta_{1}+\delta_{2}<0$ due to condition (6). The sum of the constant terms is represented by $\eta$ (which we allow to vary across countries) and - as in equation (2) - $v_{i t}$ denotes an idiosyncratic error term which reflects exogenous influences on private-sector output. ${ }^{11}$ Obviously, the key difference between the theory-based equation (8) and the standard equation (2) is that the former also includes lagged inequality, $D_{t-1}$, while the latter just ignores earlier levels of inequality. ${ }^{12}$

We are now able to analytically determine the estimate of $\delta_{1}$ if model (8) were true but the impact of inequality were estimated based on the mis-specified regression equation

$$
y_{i t}=\delta_{1} D_{i t}+\eta_{i}+w_{i t}
$$

with $w_{i t} \equiv v_{i t}+\delta_{2} D_{i t-1}$ representing the "error term". If we fit a regression line like the one in Figure 2a. (i.e., OLS based on differences), the estimated coefficient converges to $\delta_{1}-\delta_{2}(1-\pi)$ as the number of observations goes to infinity; on the other hand, if we consider a regression similar to that in Figure $2 b$. (i.e., OLS based on levels), the estimator of $\delta_{1}$ converges to $\delta_{1}+\delta_{2}(2 \pi-1)$. Note that these limits become arbitrarily close to $\delta_{1}$ and $\delta_{1}+\delta_{2}$, respectively, as $\pi$ approaches 1 . Thus, for a sufficiently large num-

\footnotetext{
${ }^{11}$ The constant $\eta_{i}$ may be country-specific due to, for instance, cross-country differences in the levels of firm productivity (even though $\left(a^{R}-a^{P}\right) / a^{R}$ is constant across countries).

${ }^{12}$ There is a simple linear relationship between the present measure of inequality, $D$, and the Gini coefficient (which is used in the empirical section). In particular, we have $G I N I=\alpha D \cdot 100$.
} 
ber of observations, the estimated coefficient approximates (but overstates) the positive short-run relationship when we rely on first differences while the level-based estimator approximates (but understates) the negative overall (long run) consequences.

To explore whether this pattern is robust to the application of more advanced estimation techniques, we go through a simple simulation exercise. In particular, we let the model generate a panel dataset consisting of observations of the type $\left(y_{i t}, D_{i t}\right)$ and then estimate the mis-specified equation (8') using the first-difference GMM and system GMM techniques (Stata commands xtabond2 y D, gmmstyle $(D)$ robust noleveleq and xtabond2 y D, gmmstyle $(D)$ robust, respectively). ${ }^{13}$ It turns out that - in qualitative terms - the results of this exercise are similar to the OLS results. In particular, we find the firstdifference GMM estimate to be positive while the system GMM estimate is negative; moreover, as is the case with the OLS estimators, the first-difference GMM approach overstates the true positive short-run effect while the system GMM estimator understates the negative overall consequences in the long run.

Stable supply of the public good. We now briefly focus on the case of stable supply of the public good. According to Proposition 1, this situation is more likely to emerge in richer economies where $a^{P}$ is sufficiently high to violate condition (7) so that $G_{t}=G_{t+1}=$ $\ldots=1$. Unless in the case of fluctuating supply, the estimation method should not affect the estimation results under these circumstances: A constant level of public investment means that $\Delta X$ is identical zero so that $\delta_{2} \equiv-\triangle X /(X(0)(H-L))=0$. As a result, the standard regression equation $\left(8^{\prime}\right)$ is the "correctly" specified empirical model.

Looking at the evidence through the lens of the model. We end this section by highlighting that our parsimonious model can "explain" important aspects of the empirical picture that emerges in Section 2 (and in the broader inequality-growth literature). On the one hand, given that the negative long-run effects are quantitatively more important

\footnotetext{
${ }^{13}$ The parameters of the model are chosen such that $\left(\delta_{1}, \delta_{2}\right)$ satisfies $\delta_{1}-\delta_{2}(1-\pi)=0.00216$ and $\delta_{1}+\delta_{2}(2 \pi-1)=-0.00198$, where 0.00216 and -0.00198 come from the OLS estimates (in differences and levels) in our full country sample (and $\pi$ is set to 0.9). Since the number of observations (OLS in levels) is about 400 (and we have maximum eight observations per country), the simulation is based on $N=50$ and $T=8$.
} 
than the positive short-run effects, the model can explain why differences-based and levelbased methods may come to opposite conclusions regarding the impact of inequality on growth. Moreover, the model tells us how to interpret the different estimation results: If within-country inequality is rather persistent (which is actually true in our dataset, see Footnote 3), the differences-based methods approximate the short-run effect while the level-based methods come close to mirroring the overall impact of inequality in the long run. On the other hand, the model also provides an explanation for the fact that there seems to be less of a discrepancy among high-income countries: In the model, more productive economies are less likely to suffer from a strong negative "long-run" effect of higher inequality. As a result, when dealing with high-income countries, the standard regression equation $\left(8^{\prime}\right)$ might be the right specification so that changes- and level-based methods must lead to similar results.

\section{Conclusions}

This paper reconciles apparently contradictory findings in the empirical literature on the inequality-growth relationship. Studies exploiting time-series variation consistently find a positive relationship. Studies exploiting also cross-sectional variation tend to find a negative link (except for the richest countries, in some cases). We use an expanded dataset confirming this empirical finding. We then introduce a simple model which allows for both positive and negative effects of inequality, and we further assume that these effects cluster in a specific way. The growth-promoting effects arise from purely economic mechanisms (convex savings, capital market imperfections, innovation incentives) and tend to set in relatively quickly, i.e., in the short or medium run. In contrast, growth-reducing effects of inequality involve the political process, the change of institutions, the rise of socio-political movements, or they operate through changes in education systems and the educational attainment of the population. Arguably, these effects take much longer and materialize primarily in the long run.

On the basis of our model, we can interpret the existing empirical results in a natural way: The differences-based estimation methods (i.e., the FE or first-difference GMM 
approaches) are likely to systematically pick up the beneficial short- or medium-run implications - and thus tend to indicate a positive relationship. The level-based methods, on the other hand, also reflect the slowly materializing (but potentially more powerful) adverse consequences of inequality; thus, the mostly negative results associated with RE or system GMM estimators should be interpreted as the overall effect of inequality in the long run. The fact that positive and the negative consequences of inequality manifest themselves at different points in time has implications for interpreting the empirical evidence. Regression equations including just one (linear) inequality term are likely to be mis-specified. According to our model, an appropriate equation should include several Gini coefficients which control for inequality at different points in the past. Clearly, the successful estimation of such equations requires long time series - and thus may become feasible in the future. 


\section{References}

[1] Acemoglu, Daron, Davide Ticchi, and Andrea Vindigni (2008); "Emergence and Persistence of Inefficient States," mimeo, MIT.

[2] Alesina, Alberto and Dani Rodrik (1994); "Distributive Politics and Economic Growth," Quarterly Journal of Economics, 109(2), 465-90.

[3] Arellano, Manuel and Stephen Bond (1991); "Some Tests of Specification for Panel Data: Monte Carlo Evidence and an Application to Employment Equations," Review of Economic Studies, 58(2), 277-297.

[4] Arellano, Manuel and Olympia Bover (1995); "Another Look at the Instrumental Variable Estimation of Error-Components Models," Journal of Econometrics, 68(1), 29-51.

[5] Atkinson, Anthony and Andrea Brandolini (2001); "Promise and Pitfalls in the Use of "Secondary" Data-Sets: Income Inequality in OECD Countries as a Case Study," Journal of Economic Literature, 39(3), 771-799.

[6] Banerjee, Abhijit and Esther Duflo (2003); "Inequality and Growth: What Can the Data Say?," Journal of Economic Growth, 8(3), 267-299.

[7] Barro, Robert (2000); "Inequality and Growth in a Panel of Countries," Journal of Economic Growth, 5(1), 5-32.

[8] Barro, Robert and Jong-Wha Lee (2001); "International Data on Educational Attainment: Updates and Implications," Oxford Economic Papers, 53(3), 541-563.

[9] Bénabou, Roland (1996); "Inequality and Growth," in B. Bernanke and J. Rotemberg, eds., NBER Macroeconomics Annual 1996, Cambridge, MA: MIT Press.

[10] Blundell, Richard and Stephen Bond (1998); "Initial Conditions and Moment Restrictions in Dynamic Panel Data Models," Journal of Econometrics, 87(1), 115-143. 
[11] Bond, Stephen, Anke Hoeffler, and Jonathan Temple (2001); "GMM Estimation of Empirical Growth Models," CEPR Discussion Papers 3048.

[12] Caselli, Francesco, Gerardo Esquivel, and Fernando Lefort (1996); "Reopening the Convergence Debate: A New Look at Cross-Country Growth Empirics," Journal of Economic Growth, 1(3), 363-389.

[13] Clarke, George (1995), "More evidence on income distribution and growth", Journal of Development Economics, 47(2), 403-427.

[14] Deininger, Klaus and Lyn Squire (1996); “A New Data Set Measuring Income Inequality," World Bank Economic Review, 10(3), 565-591.

[15] Deininger, Klaus and Lyn Squire (1998); "New ways of looking at old issues: inequality and growth," Journal of Development Economics, 57(2), 259-287.

[16] Foellmi, Reto and Josef Zweimueller (2006); "Income Distribution and DemandInduced Innovations," Review of Economic Studies, 73(4), 941-960.

[17] Foellmi, Reto and Manuel Oechslin (2008); "Why Progressive Redistribution Can Hurt the Poor," Journal of Public Economics, 92(3-4), 738-747.

[18] Forbes, Kristin (2000); "A Reassessment of the Relationship between Inequality and Growth," American Economic Review, 90(4), 869-887.

[19] Galor, Oded and Omer Moav (2004); "From Physical to Human Capital Accumulation: Inequality and the Process of Development," Review of Economic Studies, 71(4), 1001-1026.

[20] Galor, Oded and Joseph Zeira (1993); "Income Distribution and Macroeconomics," Review of Economic Studies, 60(1), 35-52.

[21] Glaeser, Edward, Jose Scheinkman, and Andrei Shleifer (2003); "The injustice of inequality," Journal of Monetary Economics, 50(1), 199-222.

[22] Hauk, William and Romain Wacziarg (2009); "A Monte Carlo Study of Growth Regressions", Journal of Economic Growth, 14(2), 103-147. 
[23] Heston, Alan, Robert Summers and Bettina Aten (2006); Penn World Table Version 6.2. Center for International Comparisons of Production, Income and Prices at the University of Pennsylvania (electronic resource).

[24] Kaldor, Nicholas (1955); "Alternative Theories of Distribution," Review of Economic Studies, 23(2), 83-100.

[25] Kuznets, Simon (1955); "Economic Growth and Income Inequality," American Economic Review, 45(1), 1-28.

[26] Li, Hongyi and Heng-fu Zou (1998); "Income Inequality is not Harmful for Growth: Theory and Evidence," Review of Development Economics, 2(3), 318-334.

[27] Mankiw, Gregory, David Romer, and David Weil (1992); "A Contribution to the Empirics of Economic Growth," Quarterly Journal of Economics, 107(2), 407-437

[28] Matsuyama, Kiminori (2000); "Endogenous Inequality," Review of Economic Studies, 67(4), 743-759.

[29] Perotti, Roberto (1993); "Political Equilibrium, Income Distribution, and Growth," Review of Economic Studies, 60(4), 755-776.

[30] Perotti, Roberto (1996); "Growth, Income Distribution, and Democracy: What the Data Say," Journal of Economic Growth, 1(2), 149-187.

[31] Persson, Torsten and Guido Tabellini (1994); "Is Inequality Harmful for Growth? Theory and Evidence," American Economic Review, 84(3), 600-621.

[32] Roodman, David (2009); "A Note on the Theme of Too Many Instruments", Oxford Bulletin of Economics and Statistics, 71(1), 135-158.

[33] Rosenzweig, Mark und Hans Binswanger (1993); "Wealth, Weather Risk and the Composition and Profitability of Agricultural Investments," Economic Journal, 103(Jan.), 56-78. 
[34] Sokoloff, Kenneth and Stanley Engerman (2000); "History Lessons: Institutions, Factors Endowments, and Paths of Development in the New World," Journal of Economic Perspectives, 14(3), 217-232.

[35] UNU-WIDER (2008); World Income Inequality Database, Version 2.0c (electronic resource).

[36] Voitchovsky, Sarah (2005); "Does the Profile of Income Inequality Matter for Economic Growth?," Journal of Economic Growth, 10(3), 273-296.

[37] World Bank (2006); World Development Indicators 2006 (electronic resource). Washington, D.C.: The World Bank. 


\section{Appendix A}

Proof of Proposition 1. (i) The first step is to introduce some notation. The value function of a representative member of group $i \in\{P, R\}$ is denoted by $V^{i}\left(D_{t}, G_{t}\right)$, whereas $D_{t}$ and $G_{t}$ are the two state variables. Thus, when thinking about the preferred level of the public good tomorrow, the poor individuals (i.e., the decisive agents) have to solve the recursive problem

$$
V^{P}\left(D_{t}, G_{t}\right)=\max _{G_{t+1} \in\{0,1\}}\left\{a^{P} \omega^{P}\left(D_{t}\right) X\left(G_{t}\right)+Z-G_{t+1} F+\beta E\left\{V^{P}\left(D_{t+1}, G_{t+1}\right)\right\}\right\} .
$$

A solution to this problem is a policy function $G_{t+1}=G^{P}\left(D_{t}, G_{t}\right)$ which gives tomorrow's level of the public good, $G_{t+1}$, as a function of the two state variables.

We now prove that - if (7) holds - the proposed policy function is in fact a solution to the recursive problem stated above (if condition 7 is violated, the equilibrium policy functions can be derived in a parallel way). To do so, we have to establish that in any given period $t$ it is indeed optimal to stick to the policy function stated in the proposition - provided that this policy function is applied in all future periods. More precisely, we have to establish that - irrespective of the value of $G_{t}$ - the representative poor agent finds it optimal to choose (i) $G_{t+1}=1$ if $D_{t}=L$ and (ii) $G_{t+1}=0$ if $D_{t}=H$ (again, provided that this rule is invariably applied in the future). The formal condition for point (i) to hold is

$$
\begin{aligned}
V^{P}\left(L, G_{t}\right) & =a^{P} \omega^{P}(L) X\left(G_{t}\right)+Z-F+\beta\left(\pi V^{P}(L, 1)+(1-\pi) V^{P}(H, 1)\right) \\
& \geq a^{P} \omega^{P}(L) X\left(G_{t}\right)+Z+\beta\left(\pi V^{P}(L, 0)+(1-\pi) V^{P}(H, 0)\right)
\end{aligned}
$$

whereas the second line in the above expression gives the value if the decision is in favor of the alternative choice, $G_{t+1}=0$. Rearranging terms yields the much simpler restriction

$$
\pi\left(V^{P}(L, 1)-V^{P}(L, 0)\right)+(1-\pi)\left(V^{P}(H, 1)-V^{P}(H, 0)\right) \geq F / \beta,
$$


which is indeed independent of $G_{t}$. Similarly, for point (ii) to be true, we must have

$$
\pi\left(V^{P}(H, 1)-V^{P}(H, 0)\right)+(1-\pi)\left(V^{P}(L, 1)-V^{P}(L, 0)\right)<F / \beta,
$$

which is again independent of the current level of the public good, $G_{t}$.

To proceed, we have to find explicit expressions for the differences $V^{P}(L, 1)-V^{P}(L, 0)$ and $V^{P}(L, 1)-V^{P}(L, 0)$ which show up in (A-1) and (A-2). Assuming that the proposed policy function is applied in all (future) periods, the two differences are given by

$$
V^{P}(D, 1)-V^{P}(D, 0)=a^{P} \omega^{P}(D)[X(1)-X(0)]
$$

with $D \in\{L, H\}$. Using this last expression in (A-1) and (A-2) completes the proof.

(ii) The second part of the proposition follows directly from condition (7). 


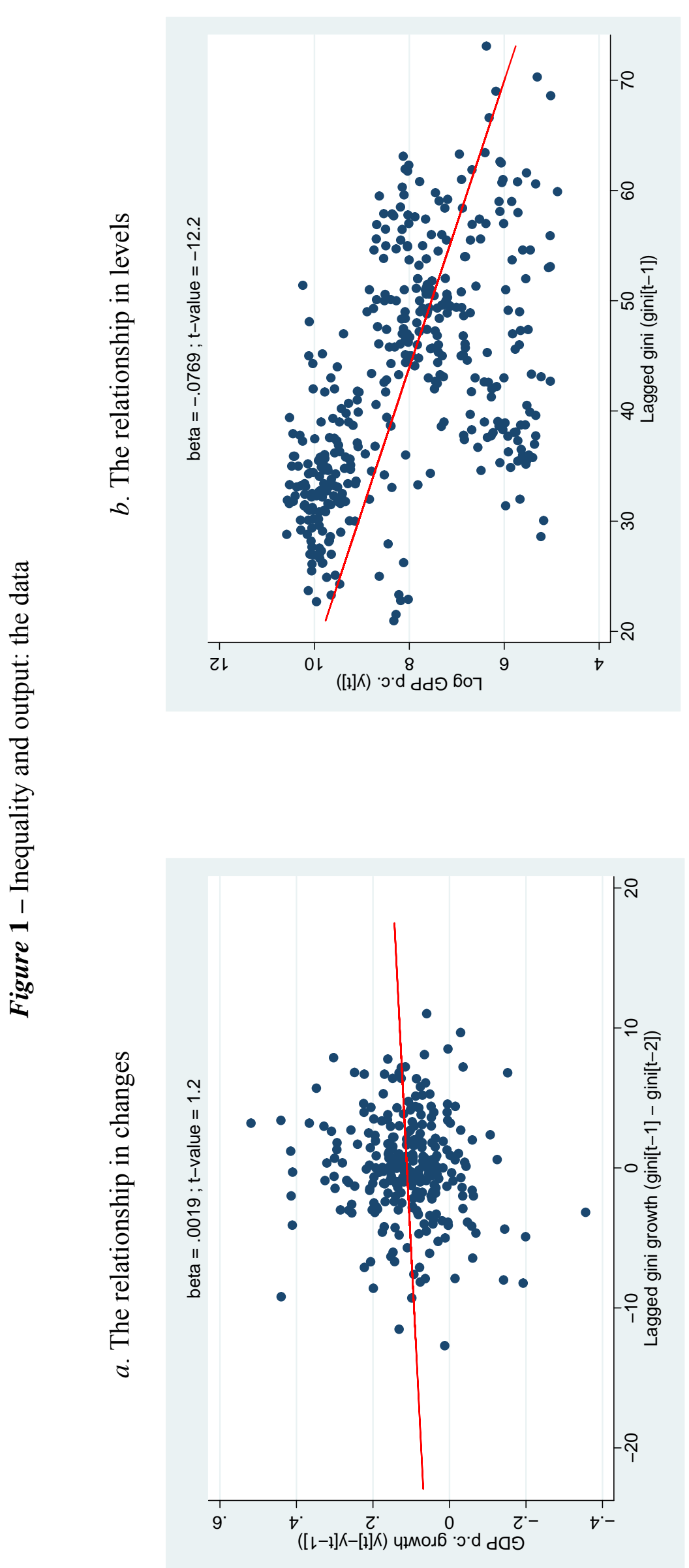

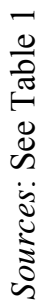




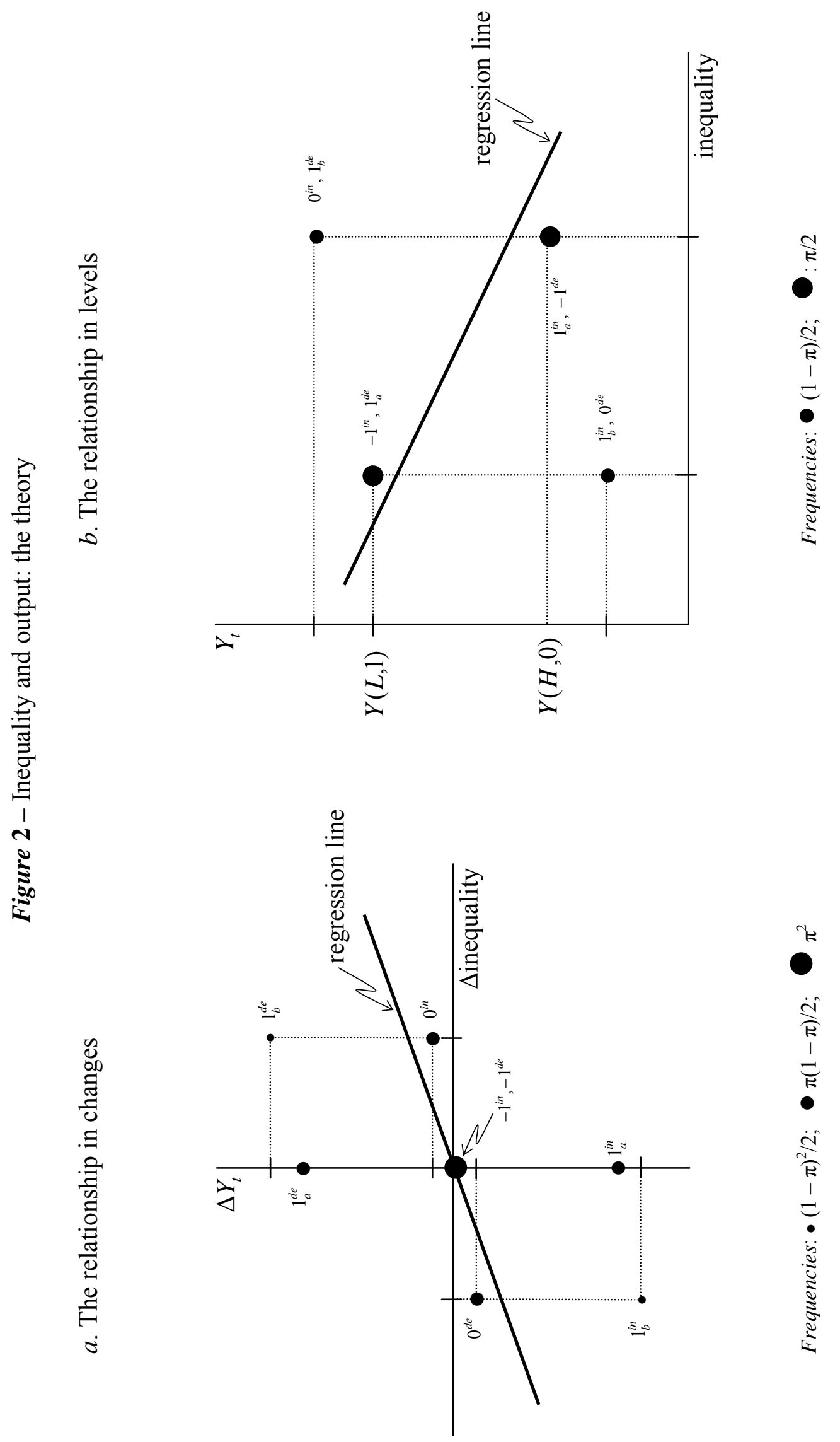




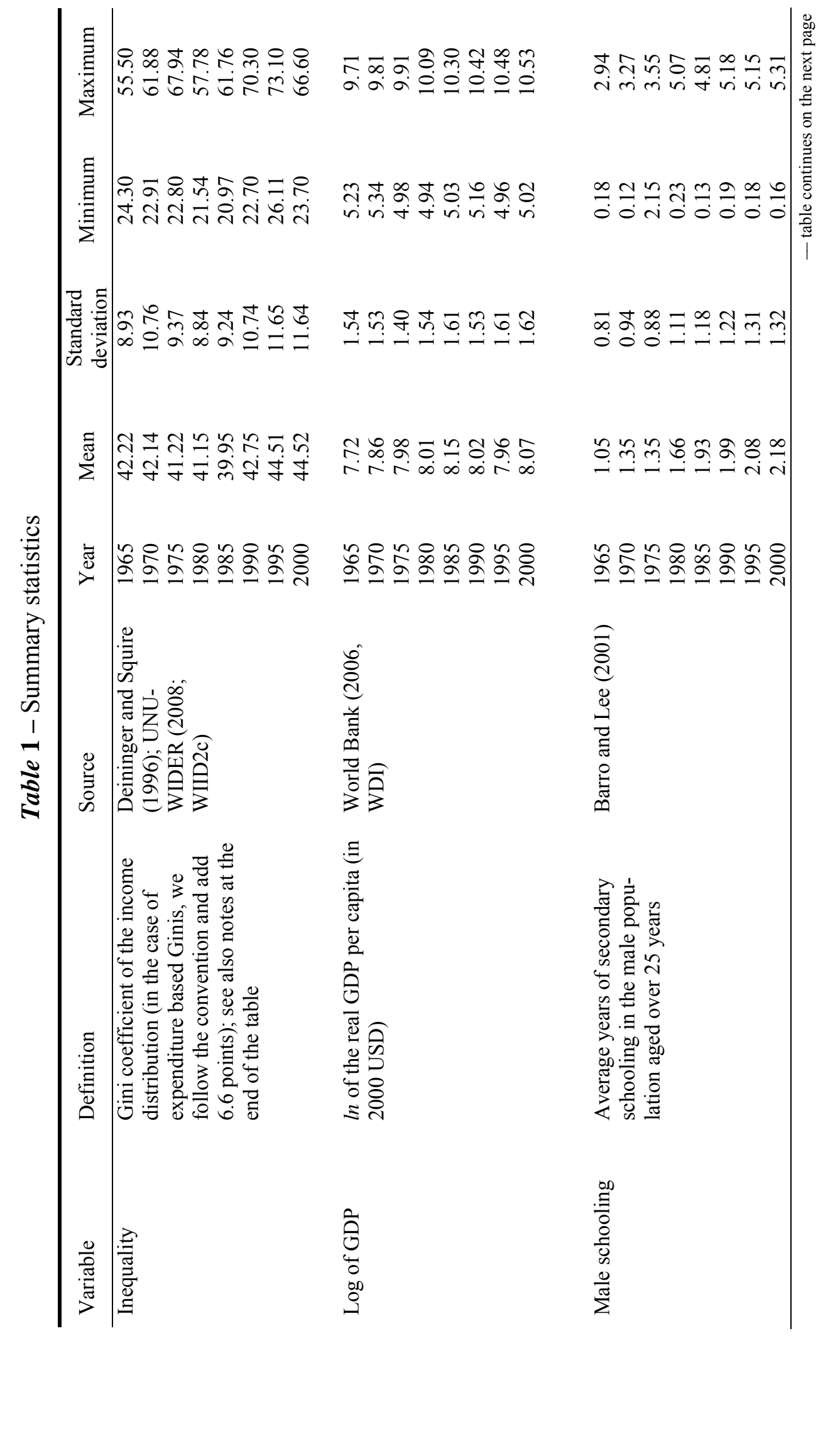




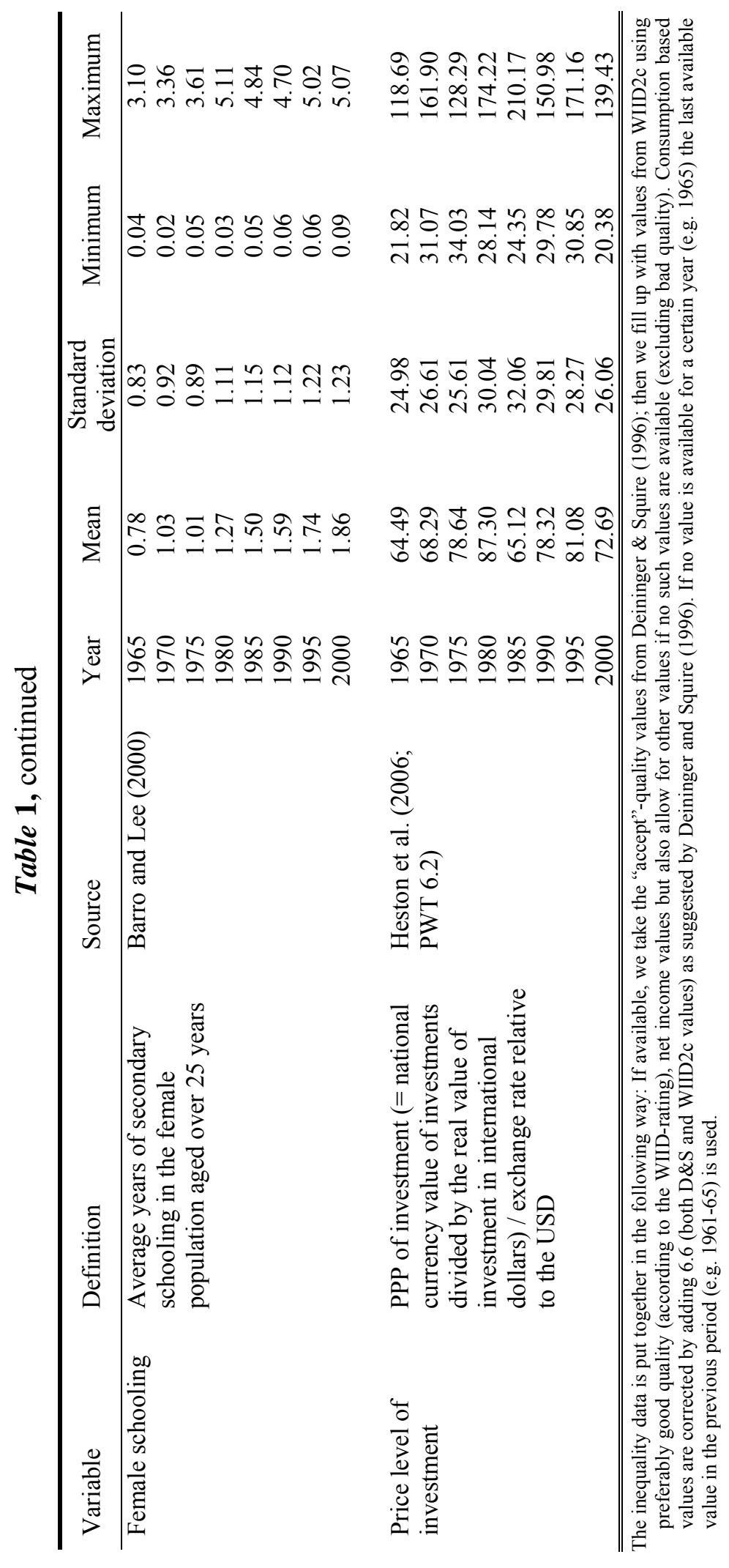




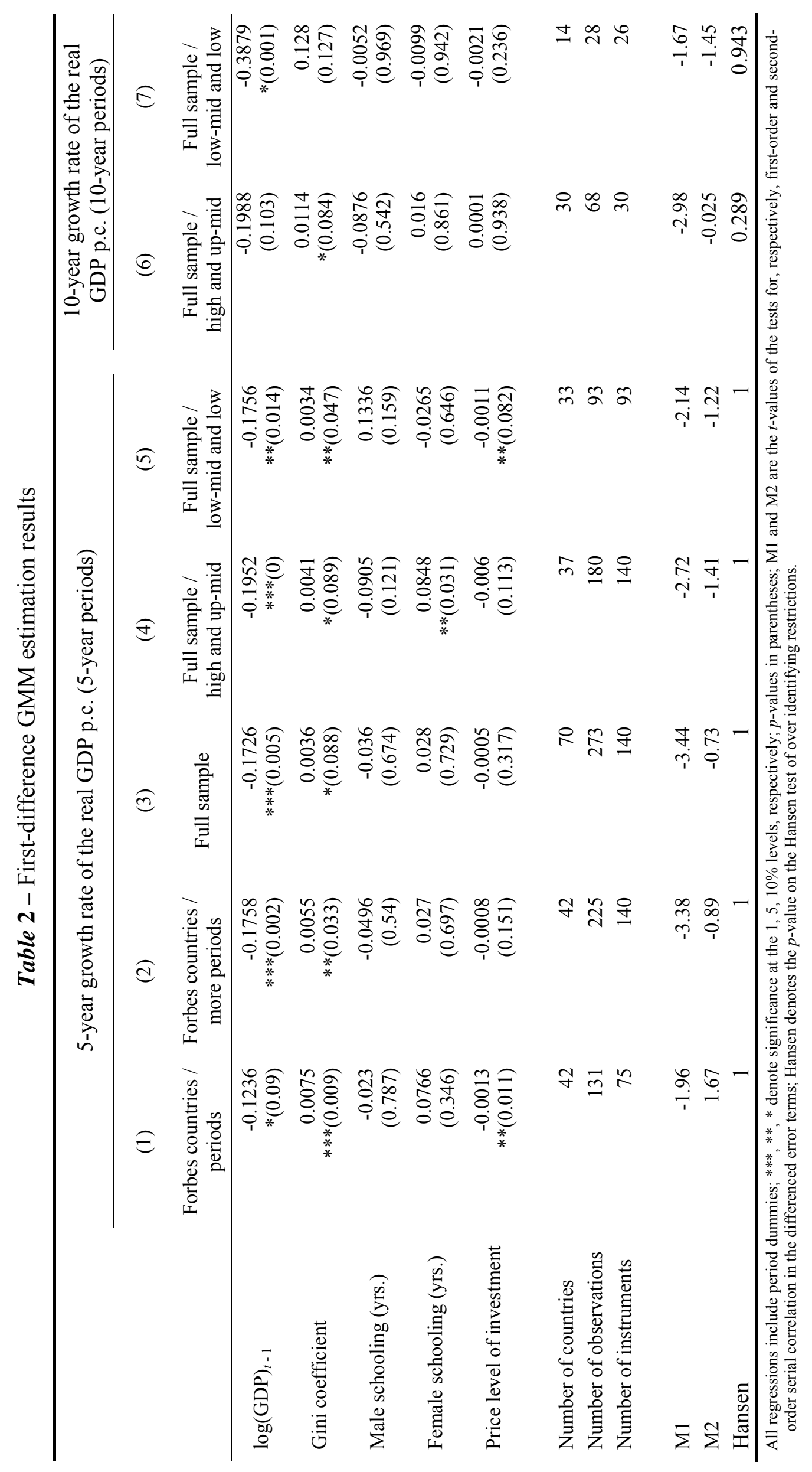




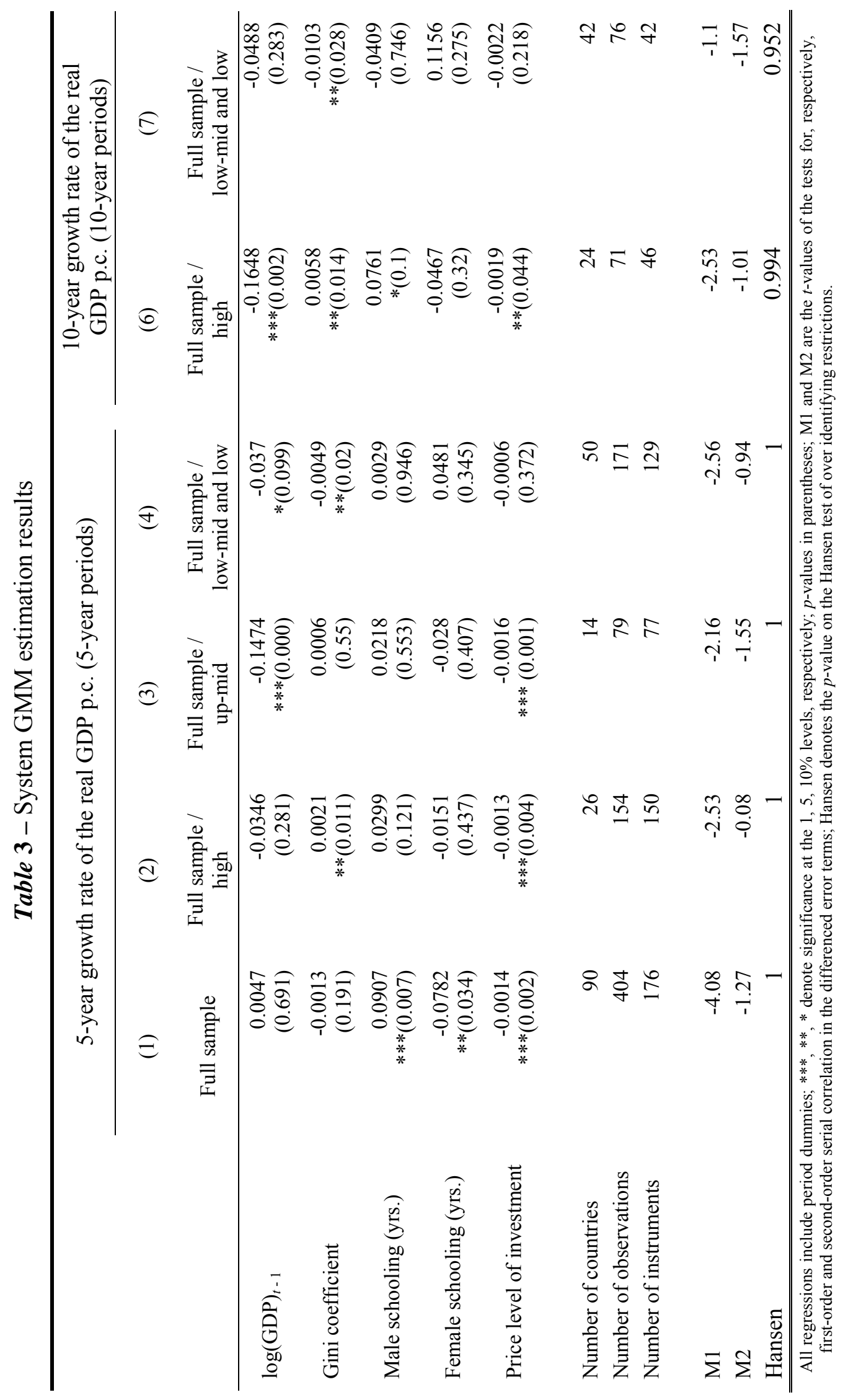




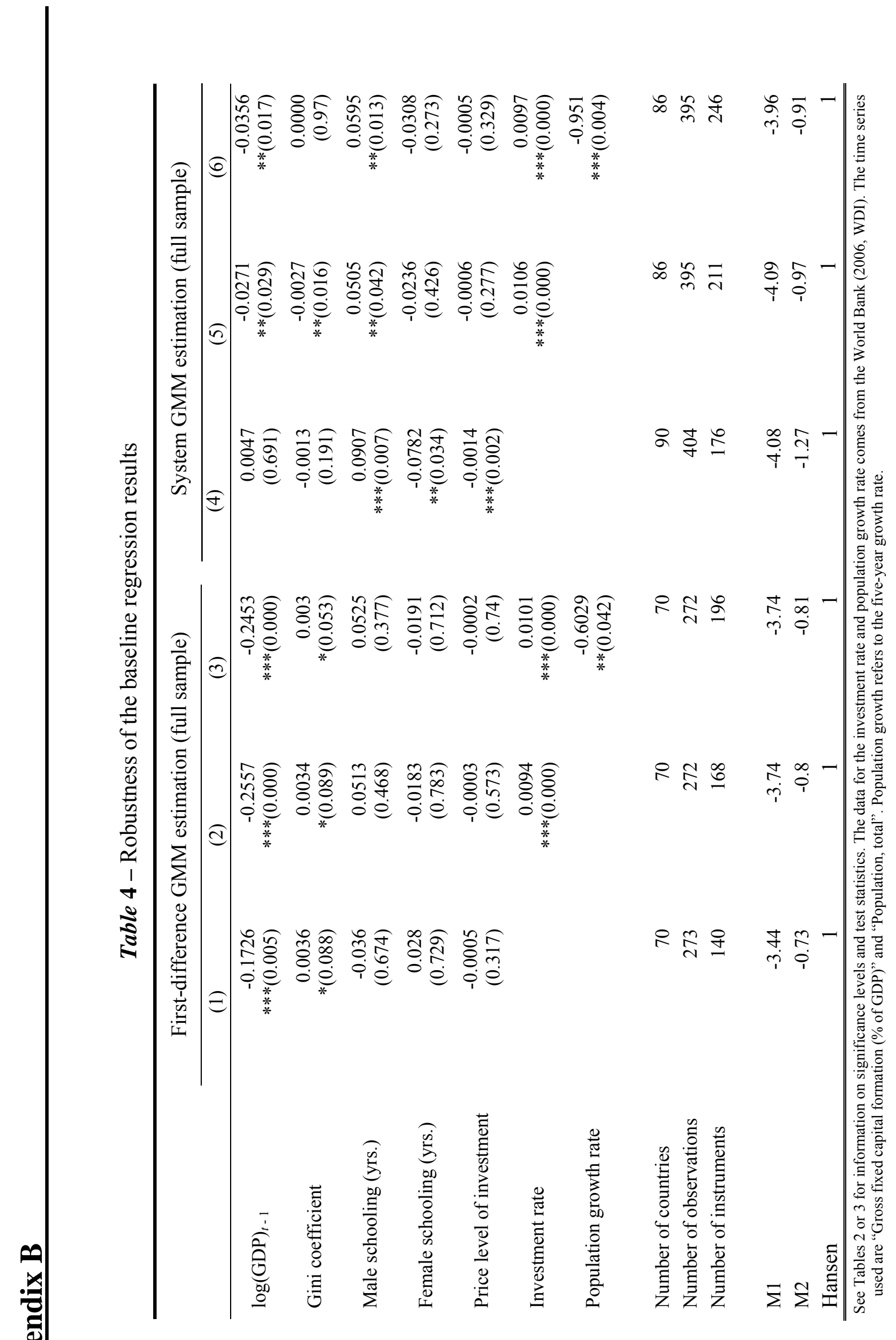

